\title{
Efecto de la densidad sobre el crecimiento y el desarrollo del petasma en langostinos juveniles Pleoticus muelleri (Decapoda, Penaeoidea)
}

\author{
Nora Harán ${ }^{1}$, Juan Mallo ${ }^{1,2}$ \& Jorge Fenucci ${ }^{1,3}$ \\ ${ }^{1}$ Departamento de Ciencias Marinas, Univ. Nacional de Mar del Plata. Funes 3350. 7600 Mar del \\ Plata. Argentina \\ ${ }^{2}$ Comisión de Investigaciones Científicas de la Provincia de Buenos Aires (CIC) \\ ${ }^{3}$ Consejo Nacional de Investigaciones Científicas y Técnicas (CONICET) \\ E-mail: nsharan@mdp.edu.ar
}

\begin{abstract}
RESUMEN. En los cultivos resultan fundamentales aspectos morfológicos y fisiológicos. Se realizó un experimento con machos juveniles de Pleoticus muelleri para comprobar el efecto de la densidad sobre el desarrollo y unión de los endópodos del primer par de pleópodos que forman el petasma. Además, se analizó el efecto de la densidad sobre el crecimiento y la supervivencia. Los ejemplares se colocaron en acuarios a dos densidades iniciales por triplicado de 14 y 28 ind $\cdot \mathrm{m}^{-2}$ y se los alimentó diariamente con manto de calamar fresco. Los pesos medios iniciales fueron 0,73 y 0,78 $\mathrm{g}$ respectivamente, y se incrementaron hasta 1,68 y 1,65 g luego de 60 días. No se encontraron diferencias significativas entre los pesos medios finales; tampoco se estableció una dependencia entre la supervivencia y los tratamientos de densidad. Ningún ejemplar presentó los endópodos unidos al iniciar el experimento, aunque se observó la presencia de pequeños ganchos que los unen entre sí (cincinnuli). Se halló una dependencia entre la densidad de ejemplares y la unión de los endópodos del primer par de pleópodos ( $80 \%$ unidos en los ejemplares con densidad inicial de 14 ind $\cdot \mathrm{m}$ ${ }^{2} \mathrm{y}$ final de $7 \mathrm{ind} \cdot \mathrm{m}^{-2}$; y $27 \%$ en los que se habían colocado a densidad inicial de $28 \mathrm{ind} \cdot \mathrm{m}^{-2}$ y final de $\left.15 \mathrm{ind} \cdot \mathrm{m}^{-2}\right)$. No se observaron diferencias significativas entre el peso medio final de los langostinos que tenían los endópodos unidos y los que no lo tenían. El crecimiento en largo del lóbulo interno del endópodo del primer par de pleópodos se ajustó a una relación lineal con el largo del cefalotórax. Adicionalmente, se estableció una relación de tipo potencial entre el largo del cefalotórax y el peso de los ejemplares en estudio.
\end{abstract}

Palabras claves: petasma, crecimiento relativo, densidad, Pleoticus muelleri.

\section{Density influence on growth and development of the petasma in juvenile shrimps Pleoticus muelleri (Decapoda, Penaeoidea)}

\begin{abstract}
In aquaculture there is considerable interest in the study of morphological and physiological aspects of shrimps under culture conditions. The petasma is a structure of the male's first pair of abdominal appendages (pleopods); the endopods of this pair join by coupling hooks (cincinnuli). It is assumed to function mechanically in transferring spermatophores. A 60 day trail was conducted with juvenile of Pleoticus muelleri to evaluate the influence of density on pleopod joint, survival and total growth. The shrimp were placed at two initial densities by triplicate (14 and 28 ind $\cdot \mathrm{m}^{-2}$ ). Survival and growth did not vary significantly between treatments. A dependence was found between density and endopod joint; no significant differences in weight were found between animals having joined and separate endopods. Relative growth studies were conducted, data inner lobe of petasmal endopod length and carapace length fit to a linear relation. Data of weight and carapace length fit to a potential relation.
\end{abstract}

Key words: petasma, relative growth, density, Pleoticus muelleri. 


\section{INTRODUCCIÓN}

El langostino argentino Pleoticus muelleri (Bate, 1888) tiene gran importancia como recurso pesquero, su área de distribución en el litoral atlántico se encuentra desde los $20^{\circ} \mathrm{S}$ en Espíritu Santo, Brasil, hasta el litoral patagónico argentino a los $50^{\circ} \mathrm{S}$, en zonas con temperaturas entre 6 y $22^{\circ} \mathrm{C}$ y salinidades de 31,5 a 33,5 psu (Boschi, 1986). Las amplias variaciones anuales y estacionales caracterizan a esta especie, por ello resulta fundamental estudiar aspectos de su biología para cultivarlos en forma independiente de la naturaleza (Fenucci et al., 1990).

Los costos de los cultivos son elevados, por lo tanto es importante establecer las condiciones que permitan reducir el tiempo de crecimiento y de maduración. Se ha estudiado el desarrollo de larvas en acuario (Scelzo \& Boschi, 1975; Boschi \& Scelzo, 1979) y diferentes condiciones de cultivo de postlarvas (Mallo \& Fenucci, 1996, 1997), así como también la cría masiva de las mismas (Mallo et al., 1999). Otros aspectos estudiados en el langostino son la maduración de hembras en cautiverio (Makinouchi et al., 1995; Díaz, 2001) y los requerimientos nutricionales (Fernández-Giménez \& Fenucci, 1998; Harán \& Fenucci, 1997a, 1997b).

En general, los crustáceos decápodos son agresivos y presentan territorialidad (HoltschmitMartínez, 1987). Además, en algunas especies se ha comprobado la existencia de feromonas que afectan el crecimiento (Salmeron, 1985, en HoltschmitMartínez, 1987). Estos fenómenos tienen especial importancia en los sistemas de cultivo donde los organismos se encuentran confinados, y en muchos casos dificultan el mantenimiento de ejemplares en espacios reducidos. La densidad a la que se encuentran los individuos puede influir tanto sobre el aumento del tamaño total como sobre algún proceso en su desarrollo. Un aspecto para analizar es la madurez morfológica, en la que se unen las porciones izquierda y derecha del petasma; ésta es un hito de transición entre las fases de juvenil y subadulto, en la cual se completará la formación de las glándulas sexuales (García, 1977).

En los camarones peneidos, los endópodos de los pleópodos 1 y 2 de los machos están modificados: los endópodos de los pleópodos 1 de cada lado articulan entre sí por medio de pequeños ganchos (cincinnuli) para formar el petasma, con formas variables en las distintas especies de peneidos; a veces el endópodo del pleópodo 2 porta el appendix masculina; estas dos estructuras intervienen en la transferencia del espermatóforo a la hembra; el petasma varía entre las especies según su grado de apertura ventral y en la presencia de cuernos o ganchos distales, por lo que estas estructuras tienen importancia en la sistemática de estos crustáceos (Bauer, 1991).

En P. muelleri cada endopodito petasmal se compone de una parte membranosa flexible y de otra rígida; esta última forma el armazón del sistema y al mismo tiempo es la unión de la membrana petasmal con el basipodito; la membrana petasmal se divide en dos lóbulos: uno interno o mediano y otro externo o lateral (Boschi \& Angelescu, 1962). Guitart et al. (1985) señalan que en las especies Lithopenaeus schmitti y Farfantepenaeus notialis los endópodos del primer par de pleópodos aumentan de tamaño y se unen en la fase adulta para formar el petasma.

Se realizó un experimento con machos juveniles de $P$. muelleri para estudiar la influencia de la densidad en el desarrollo y unión de los endópodos del primer par de pleópodos, también el efecto de la densidad sobre el crecimiento y la supervivencia de los langostinos. Paralelamente se hicieron mediciones del largo del cefalotórax y del largo del lóbulo interno del endópodo petasmal de los ejemplares y se buscó una correlación entre el peso y el largo del cefalotórax de estos ejemplares.

\section{MATERIALES Y MÉTODOS}

El experimento se llevó a cabo utilizando machos juveniles de langostino Pleoticus muelleri de peso medio inicial 0,50-1,28 g provenientes de una larvicultura realizada según la técnica descripta por Mallo et al. (1999) en la Estación Nágera dependiente del Departamento de Ciencias Marinas (Universidad Nacional de Mar del Plata), ubicada en Chapadmalal, Provincia de Buenos Aires, Argentina.

Se utilizaron acuarios de 150 litros con filtro biológico interno y sustrato de arena y conchillas (Boschi, 1972). Los ejemplares se pesaron en una balanza digital (precisión 0,001 g) y se dividieron en dos grupos: un grupo de 21 ejemplares se colocó a densidad inicial de $14 \mathrm{ind} \cdot \mathrm{m}^{-2}$ (peso medio $0,73 \pm$ $0,156 \mathrm{~g}$ ) y el otro de 42 ejemplares se ubicó a una densidad inicial de $28 \mathrm{ind} \cdot \mathrm{m}^{-2}$ (peso medio $0,78 \pm$ $0,209 \mathrm{~g}$ ), cada tratamiento se realizó por triplicado. Durante 60 días se alimentaron con manto de cala- 
mar fresco. En ese periodo la temperatura del agua osciló entre 17 y $19^{\circ} \mathrm{C}$, la salinidad se mantuvo constante a 33 psu y el pH varió entre 7,0-7,5.

Al inicio del experimento ningún ejemplar tenía unidos entre sí los endópodos del primer par de pleópodos, aunque ya estaban formados los cincinnuli, los cuales se hallan implantados en varias hileras y cada uno tiene forma de un doble gancho (Fig. 1).

Al inicio y al final del experimento se midió el cefalotórax con microscopio estereoscópico provisto de un ocular micrométrico graduado, se consideró la distancia desde la foseta postorbital al extremo dorsal posterior del cefalotórax. También, al inicio y al final se midieron los lóbulos internos de los endópodos petasmales desde su base hasta el extremo distal tal como se indica en la Figura 2.

Al finalizar el experimento se tomaron fotografías con un microscopio con cámara automática reflex, a diferentes aumentos para observar con mayor detalle las estructuras que forman el petasma (Fig. 3). Se analizaron los datos obtenidos utilizando los siguientes tests estadísticos ( $\mathrm{p} \leq$ 0,05): test de homoscedasticidad de varianzas, $t$ de Student para diferencias de peso medio, $\chi^{2}$ para detectar independencia de atributos entre densidad y supervivencia, y entre la densidad y la unión de los endópodos. Además, se realizó un análisis de regresión en el caso del crecimiento relativo entre el largo del cefalotórax y el peso, y entre el largo del cefalotórax y el largo del lóbulo interno del pleópodo petasmal, en este último caso se compararon las rectas de regresión halladas al medir los ejemplares bajo los dos tratamientos de densidad (Sokal \& Rohlf, 1981).

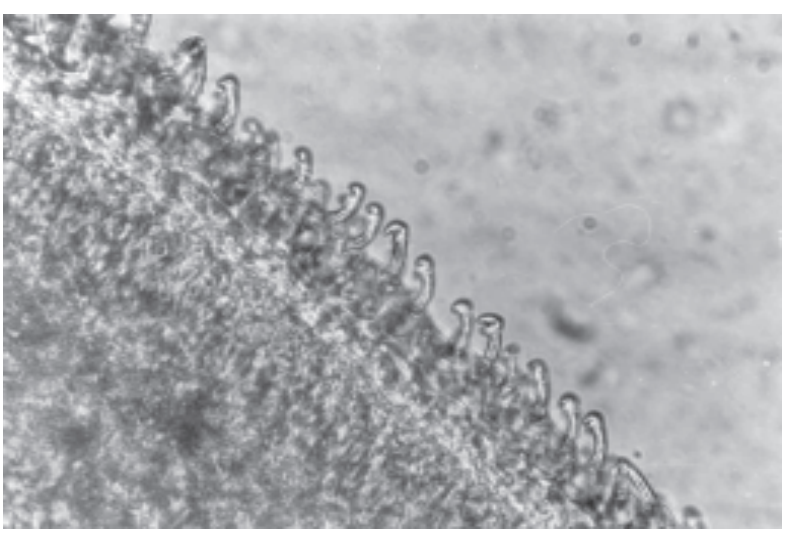

Figura 1. Ganchos de unión (cincinnuli) ubicados en el borde interno del endópodo petasmal de los machos juveniles de $\boldsymbol{P}$. muelleri.

Figure 1. Coupling hooks (cincinnuli) in the inner border of petasmal endopods in juvenile males of $P$. muelleri.

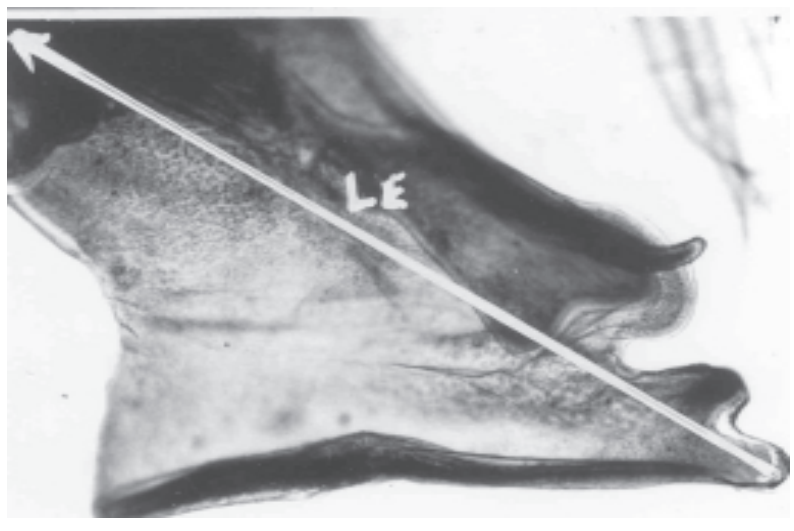

Figura 2. Endópodo del primer par de pleópodos de macho juvenil de $P$. muelleri. $\mathrm{L}_{\mathrm{E}}$ : línea de medición de la longitud del endópodo.

Figure 2. Endopod of the first pleopod in juvenile male of $P$. muelleri. $L_{E}$ : measuring line of the endopod lenght.

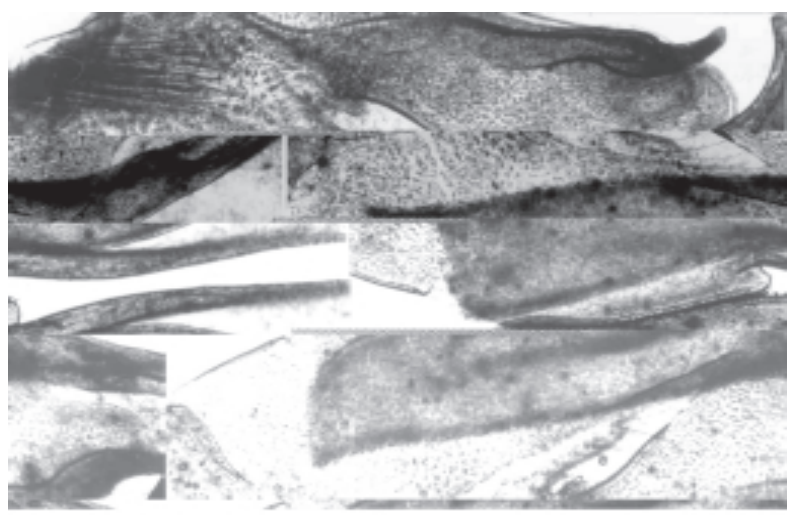

Figura 3. Vista del endópodo petasmal de machos juveniles de $P$. muelleri.

Figure 3. View of the petasmal endopod in juvenile males of $P$. muelleri. 


\section{RESULTADOS}

Los valores iniciales y finales de densidad y peso medio de los ejemplares machos juveniles de Pleoticus muelleri se indican en la Tabla 1. El mayor incremento del peso medio (130\%) se registró en los langostinos que se colocaron a densidad inicial de $14 \mathrm{ind} \cdot \mathrm{m}^{-2} \mathrm{y}$ en el otro tratamiento el incremento fue de $112 \%$, no se encontraron diferencias significativas entre los pesos medios finales de ambos tratamientos $(\mathrm{p} \leq 0,05)$. Tampoco se halló dependencia con los tratamientos de densidad al comparar los valores de supervivencia de los langostinos (Fig. 4). La mortalidad se debió fundamentalmente al canibalismo que presentó esta especie principalmente durante la exuviación y antes del endurecimiento del nuevo exoesqueleto.

Se halló una dependencia entre la densidad de los ejemplares y la unión de los endópodos del primer par de pleópodos. Al final del experimento, el porcentaje de unión para el tratamiento de menor densidad tuvo un valor de $80 \%$ y el del tratamiento de mayor densidad fue de $27 \%$ (Fig. 4).

No hubo diferencias significativas entre el peso medio de los individuos que tenían los endópodos del primer pleópodo unidos $(1,58 \pm 0,386 \mathrm{~g})$ y los que no los tenían unidos $(1,70 \pm 0,372 \mathrm{~g})$.
Se determinó la existencia de una relación lineal $(\mathrm{p} \leq 0,05)$ entre el largo de los endópodos del primer par de pleópodos y el largo del cefalotórax; previamente se compararon rectas de regresión surgidas de los datos obtenidos en cada uno de los tratamientos de densidad, no encontrándose diferencias entre las pendientes, debido a ello los datos se presentaron en una sola recta (Fig. 5).

La tasa de crecimiento constante en el largo del cefalotórax y en el largo del endópodo correspondió a un crecimiento isométrico. En la Figura 6 se observa una relación potencial entre el largo del cefalotórax y el peso de los individuos ( $\mathrm{p} \leq 0,05$ ), correspondiente a un crecimiento de tipo alométrico positivo ya que la constante de crecimiento tuvo un valor de 3,01 (Hartnoll, 1982).

Tabla 1. Pesos medios iniciales y finales en machos juveniles de Pleoticus muelleri bajo dos tratamientos de densidad.

Table 1. Initial and final mean weight in juvenile male of Pleoticus muelleri at two density treatments.

\begin{tabular}{|cc|cc|}
\hline \multicolumn{2}{|c|}{ Densidad $\left(\right.$ ind $\cdot \mathbf{m}^{-2}$ ) } & \multicolumn{2}{|c|}{ Peso medio $(\mathbf{g})$} \\
\hline Inicial & Final & Inicial & Final \\
14 & 7 & $0,74 \pm 0,024$ & $1,68 \pm 0,173$ \\
28 & 15 & $0,78 \pm 0,044$ & $1,65 \pm 0,141$ \\
\hline
\end{tabular}

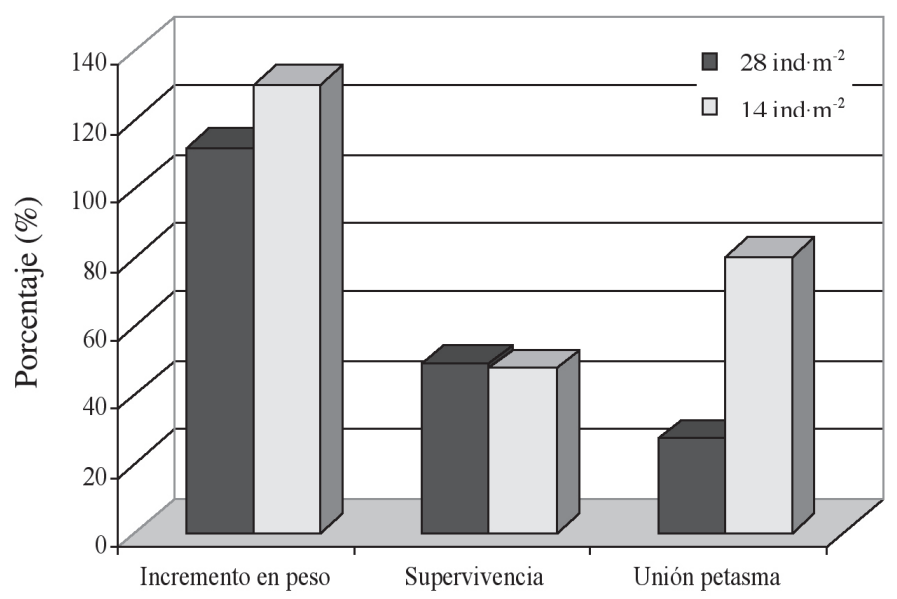

Figura 4. Incremento en peso, supervivencia y unión del petasma en machos juveniles de $P$. muelleri bajo dos tratamientos de densidad.

Figure 4. Weight raise, survival and petasmal joint in juvenile male of $P$. muelleri at two density treatments. 


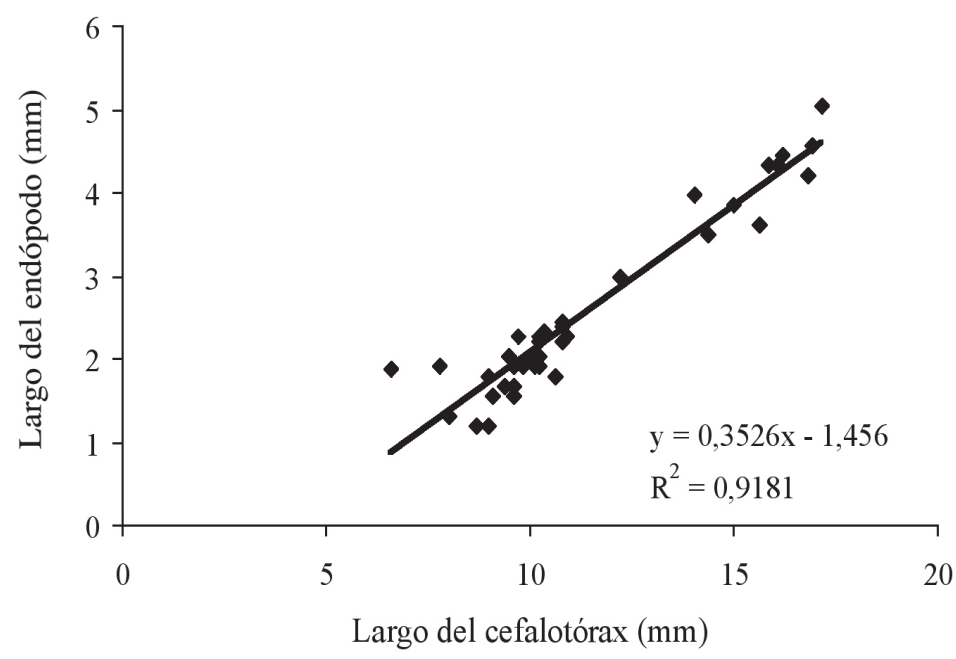

Figura 5. Crecimiento relativo del langostino P. muelleri. Relación entre la longitud del endópodo petasmal y el largo del cefalotórax en los machos juveniles.

Figure 5. Relative growth of the shrimp $P$. muelleri. Relationship between the petasmal endopod length and the carapace length of juvenile males.

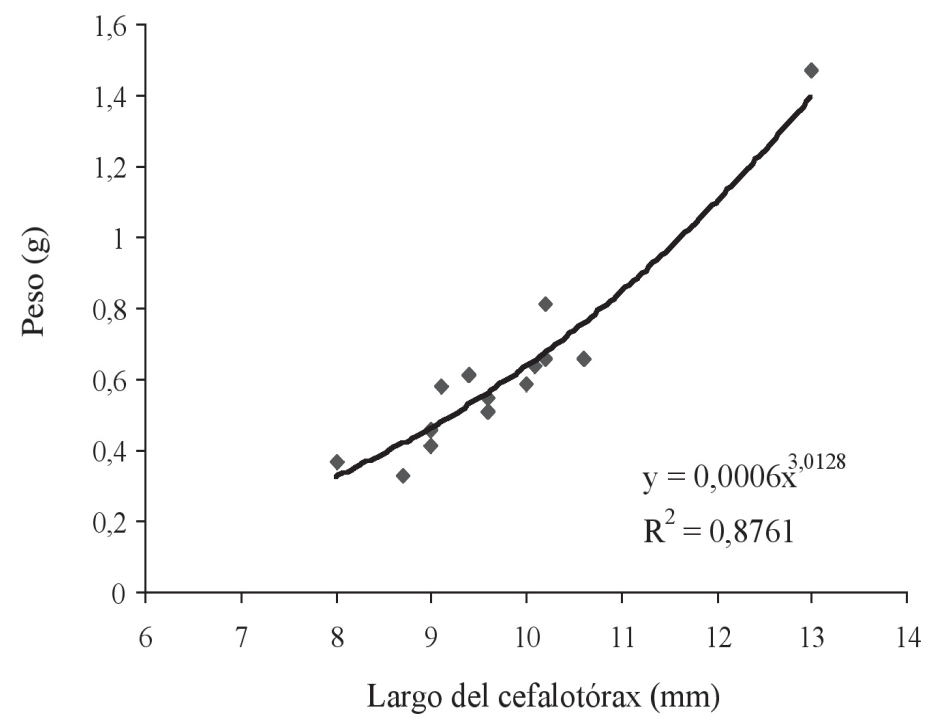

Figura 6. Crecimiento relativo del langostino $P$. muelleri. Relación entre el peso y el largo del cefalotórax en los machos juveniles.

Figure 6. Relative growth of the shrimp $P$. muelleri. Relationship between the weight and the carapace length of juvenile males. 


\section{DISCUSIÓN}

En general, la dominancia implica cierta jerarquía donde los organismos más grandes tienen prioridad sobre el alimento, refugio o pareja sexual. El aspecto más relevante de la territorialidad es que aunado a las interacciones sociales crea un efecto de inhibición del crecimiento que se acentúa cuanto mayor es la densidad de la población (Salmeron, 1985). Nelson et al. (1983) demostraron que la inhibición del crecimiento de langostas juveniles del género Homarus es debida a la influencia de individuos de mayor edad, y ésta decae rápidamente con la distancia; la mayor ganancia en peso se logra en sistemas que minimizan la inhibición del crecimiento al utilizar ejemplares de tallas semejantes. En este experimento, en que se utilizaron individuos de tamaños similares, no se observó un efecto de la densidad sobre el crecimiento de los machos juveniles de P. muelleri, ya que luego de 60 días no se hallaron diferencias entre los pesos medios finales de ambos tratamientos y no se observó gran dispersión de esa variable.

Debido a que no se encontraron diferencias estadísticamente significativas entre los pesos medios de los langostinos que tenían los endópodos del petasma unidos y los que no los tenían unidos, el tamaño de los ejemplares no sería el determinante en la unión.

Un aspecto básico para los cultivos es la identificación de los sexos. En el caso de los carideos (Macrobrachium rosenbergii) existe un dimorfismo sexual y es fácil distinguir los machos de las hembras, principalmente por el tamaño y las quelas (Holtschmit-Martínez, 1987). La identificación en los peneidos se hace principalmente observando el primer par de pleópodos. En el caso de P. muelleri, las principales características del dimorfismo sexual masculino se evidencian por las modificaciones en el coxopodito del quinto par de pereiópodos, el petasma y el appendix masculina (Boschi \& Angelescu, 1962). El petasma está formado por los endopoditos del primer par de pleópodos que en los machos adultos se unen sobre el borde membranoso interno mediante los cincinulli. Yin et al. (1986) observaron la diferenciación de los órganos genitales externos de Penaeus orientalis a partir de los primeros estadios postlarvales. Los caracteres sexuales secundarios de Lithopenaeus schmitti y Farfantepenaeus notialis comienzan a desarrollar- se al final de la fase juvenil, marcando el inicio de la fase de preadulto; los endópodos del primer par de pleópodos aumentan de tamaño y en la fase adulta se unen formando el petasma (Guitart et al., 1985).

En este experimento se observó que en $P$. muelleri los caracteres sexuales secundarios aparecen en las postlarvas, y además que el fenómeno de la unión del petasma en los juveniles estaría relacionada con la densidad a la que se encuentran, ya que es diferente el porcentaje de unión en organismos que se encuentran a densidades distintas. Se hace necesario continuar con los estudios para establecer las causas fisiológicas por las que se observan diferentes porcentajes de unión.

En los estudios de crecimiento relativo se comparan las relaciones de dos dimensiones en el tiempo, una de ellas es la que representa el tamaño general del animal y la otra representa el crecimiento relativo que se busca describir. En algunos casos, estos estudios son importantes en la identificación de especies (Kitani, 1992), o bien permite inferir diferentes condiciones de vida, tal es el caso del distinto crecimiento relativo del carpo de postlarvas de cultivo y salvajes de Lithopenaeus vannamei (Kitani, 1993). Las rectas que surgen del análisis de los datos obtenidos bajo los dos tratamientos de densidad, que representan diferentes condiciones ambientales, no son significativamente distintas, por lo tanto se describe con una única recta el desarrollo del endópodo del primer par de pleópodos con respecto al largo del cefalotórax de machos juveniles de cultivo de $P$. muelleri.

En la población natural de $P$. muelleri, con muestras obtenidas con una red de arrastre en el área costera de Mar del Plata, Argentina $\left(38^{\circ} \mathrm{S}\right)$, la relación entre el peso y largo del cefalotórax se ajusta a una regresión potencial tanto en hembras como en machos sin diferencias entre sexos y los valores de la constante de crecimiento alométrico varían entre 2,62 y 2,64 (Díaz, 2001). Cabe destacar que en las muestras de la población no se consideraron individuos menores a $3 \mathrm{~g}$ debido a la selectividad del arte de pesca. Con datos de adultos de la misma zona, Angelescu \& Boschi (1959) obtuvieron un valor de la constante de crecimiento de 3,20. En el caso de los machos juveniles, en el rango de tamaños analizado, la constante de crecimiento es 3,01, valor que se encuentra entre los observados en los adultos salvajes. 


\section{CONCLUSIONES}

1. Se comprobó que la unión del primer par de pleópodos de los machos para formar el petasma es dependiente de la densidad a las que se ubicaron los machos juveniles de P. muelleri (14 y 28 ind $\cdot \mathrm{m}^{-2}$ ).

2. No se observó efecto de dichas densidades sobre el crecimiento en peso ni sobre la supervivencia.

3. No se hallaron diferencias significativas entre el peso medio de los ejemplares que tenían los endópodos unidos y los que no lo tenían.

4. Se estableció una relación lineal entre largo del endópodo del primer pleópodo de los machos juveniles de esta especie y el largo del cefalotórax. También, se halló una relación potencial entre el largo del cefalotórax y el peso, estas relaciones caracterizan a los ejemplares en esta etapa de su ciclo de vida y en las condiciones de cultivo descriptas.

\section{AGRADECIMIENTOS}

Los autores agradecen el financiamiento de la Agencia de Promoción Científica y Tecnológica y la Universidad de Mar del Plata, Argentina, en el marco del Proyecto PICT 2000-2001 № 8-8615.

\section{REFERENCIAS}

Bauer, R. 1991. Sperm transfer and storage structures in penaeoid shrimps: a functional and phylogenetic perspective. En: R.T. Bauer \& J.W. Martin (eds.). Crustacean sexual biology. New York, pp. 183-207.

Boschi, E. 1972. El acuario de agua salada. Contrib. Inst. Biol. Mar., Mar del Plata, 220: 23 pp.

Boschi, E. 1986. La pesquería del langostino del litoral patagónico. Cuadernos de Redes, Buenos Aires, 20: $8 \mathrm{pp}$.

Boschi, E. \& V. Angelescu. 1962. Descripción de la morfología externa e interna del langostino con algunas aplicaciones de índole taxonómico y biológico (Hymenopenaeus mülleri) Bate Crustacea, Fam. Penaeidae. Bol. Inst. Biol. Mar., Mar del Plata, 1: 1-75.

Boschi, E. \& M. Scelzo. 1979. El cultivo de camarones comerciales peneidos en la Argentina y la posibilidad de su producción en mayor escala. En: T. Pillary, \& W. Dill (eds.). Advances in aquaculture.
FAO Technical Conference on Aquaculture, Kyoto, Fishing News Books Ltd, Surrey, pp. 268-270.

Díaz, A. 2001. Estudio sobre la fisiología de la muda y la maduración gonadal del langostino Pleoticus muelleri Bate. Tesis Doctoral. Departamento de Ciencias Marinas, Facultad de Ciencias Exactas y Naturales, Universidad Nacional de Mar del Plata, $171 \mathrm{pp}$.

Díaz, A. \& J. Fenucci. 2002. Comparative evaluation of different animal protein source in juveniles of Pleoticus muelleri (Crustacea, Penaoidea). En E. Escobar-Briones \& F. Alvarez (eds.). Modern approaches to the study of Crustacea. Kluwer Academic/Plenum Publishers, New York, pp. 75-78.

Díaz, A., A. Fernández-Giménez \& J. Fenucci. 1999. Evaluación del extracto proteico de calamar en la nutrición del langostino argentino Pleoticus muelleri Bate (Decapoda, Penaeoidea). Mem. Acuicult. Venezuela, 99(1): 184-192.

Fenucci, J., M. Muller \& J. Magnaterra. 1990. Factibilidad de cría del langostino (Pleoticus muelleri). Frente Marítimo, 7B: 103-108.

Fernández-Giménez, A. \& J. Fenucci. 1998. Alimentación del langostino Pleoticus muelleri Bate, (Decapoda, Penaeoidea) con distintos niveles de vitamina E. Influencia sobre el crecimiento y la supervivencia. Aquicultura Brasil, 98(2): 49-54.

García, S. 1977. Biologie et dynamique des populations de crevettes roses, Penaeus duorarum notialis (Perez-Farfante, 1967) en Cote d'Ivoire. Trav. Doc. ORSTOM, 79: 1-271.

Guitart, E., E. González, R. Reyes \& I. Fraga. 1985. Descripción del aparato reproductor masculino de Penaeus notialis y Penaeus schmitti. Rev. Cub. Inv. Pesq., La Habana, 10(1-3): 41-58.

Harán, N. \& J. Fenucci. 1997a. Acción de ácidos grasos dietarios sobre el crecimiento del langostino argentino Pleoticus muelleri Bate. Rev. Invest. Mar. (Univ. de La Habana-Univ. Nac. Autónoma de México), 18(2): 155-161.

Harán, N. \& J. Fenucci. 1997b. Efecto del colesterol en la dieta del langostino argentino Pleoticus muelleri Bate. Rev. Cub. Inv. Pesq., La Habana, 20(2): 40-43.

Hartnoll, R. 1982. Growth. En: L.G. Abele (ed.). The Biology of Crustacea. Academic Press, New York, 2:111-185. 
Holtschmit-Martínez, K. 1987. Manual técnico para el cultivo y engorda del langostino malayo. Fondepesca, Monterrey, 128 pp.

Kitani, H. 1992. Relative growth of penaeid postlarvae as a useful tool for identification. Nippon Suisan Gakkaishi, 58(12): 2211-2217.

Kitani, H. 1993. Morphology of postlarvae of the whiteleg shrimp Penaeus vannamei. Nippon Suisan Gakkaishi, 59(2): 223-227.

Makinouchi, S., E. Sarlo, C. Díaz \& M. Alvarez. 1995. Maturation, spawning, molting cycle and growth of eyestalk-ablated and unablated Argentine red shrimp Pleoticus muelleri (Decapoda, Solenoceridae). Suisanzoshoku, H7: 331-336.

Mallo, J. \& J. Fenucci. 1996. Crecimiento y supervivencia de postlarvas del langostino Pleoticus muelleri con dos dietas y diferentes densidades de siembra. Rev. Cub. Inv. Pesq., La Habana, 20(2): 50-54.

Mallo, J. \& J. Fenucci. 1997. Crecimiento y supervivencia de postlarvas de langostino Pleoticus muelleri a diferentes salinidades. Rev. Cub. Inv. Pesq., La Habana, 21(1): 36-40.

Mallo, J., J. Fenucci \& C. Galarza. 1999. Cría masiva de larvas y postlarvas del langostino argentino

Recibido: 11 junio 2003; Aceptado: 16 enero 2004
Pleoticus muelleri Bate (Crustacea, Decapoda, Solenoceridae). Mem. Acuicult. Venezuela, 99(1): 318-327.

Nelson, K., D. Hedgecock, B. Heyer \& T. Nunn. 1983. On the nature of short-range growth inhibition in juvenile lobsters (Homarus americanus). J. Exp. Mar. Biol. Ecol., 72: 83-98.

Salmeron, J. 1985. Efectos de la densidad, interacción química, interacción visual, preferencia de color y efecto de color de sustrato en el crecimiento de postlarvas del langostino Macrobrachium rosenbergii (De Man). Tesis de Maestría I.T.E.S.M. Guaymas, México, 186 pp.

Scelzo, M. \& E. Boschi. 1975. Cultivo del langostino Hymenopenaeus muelleri (Crustacea, Decapoda, Penaeidae). Physis, Buenos Aires, Secc. A, 34(88): 193-197.

Sokal, R. \& J. Rohlf. 1981. Biometry. The principles and practice of statistics in biological research. W.H. Freeman \& Co., San Francisco, 776 pp.

Yin, Z., W. Song, L. Ma \& J. Yu. 1986. Studies on the development and differentiation of external genital organs of Penaeus orientalis. Trans. Oceanol. Limnol. Haiyang Huzhao Tongbao, 4: 5661. 